\title{
Compound heterozygosity for KLF1 mutations is associated with microcytic hypochromic anemia and increased fetal hemoglobin
}

\author{
Jiwei Huang ${ }^{1,3}$, Xinhua Zhang, ${ }^{2,3}$, Dun Liu ${ }^{1}$, Xiaofeng Wei ${ }^{1}$, Xuan Shang ${ }^{1}$ Fu Xiong ${ }^{1}$, Lihua Yu1, Xiaolin Yin ${ }^{2}$ \\ and Xiangmin $\mathrm{Xu}^{\star, 1}$
}

Krüppel-like factor 1 (KLF1) regulates erythroid lineage commitment, globin switching, and the terminal maturation of red blood cells. Variants in human KLF1 have been identified as an important causative factor in a wide spectrum of phenotypes. This study investigated two unrelated male children in China who had refractory anemia associated with poikilocythemia. These were accompanied by an upregulation of biochemical markers of hemolysis, along with abnormal hemoglobin $(\mathrm{Hb})$ level and elevated reticulocyte counts. Next-generation sequencing revealed that the patients were compound heterozygotes for a $K L F 1$ frameshift mutation c.525_526insCGGCGCC (p.(Gly176ArgfsTer179)) and one of two missense variants, c.892 G > C (p.(Ala298Pro)) and c.1012C $>$ T (p.(Pro338Ser)). The subjects had microcytic hypochromic anemia, and their healthy parents had single mutation. The two missense mutations affected a highly conserved codon in the zinc finger DNA-binding domain of KLF1, but the protein stability was unaffected in K-562 cells. A KLF1-targeted promoter-reporter assay showed that the two mutations reduce the expression of the $H B B, B C L 11 A$, and $C D 44$ genes involved in erythropoiesis, with consequent dyserythropoiesis and an $\alpha /$ non- $\alpha$ chain imbalance. A systematic analysis was performed of the phenotypes associated with the KLF1 mutations in the two families, and the clinical characteristics and differential diagnoses of the disease are presented. This is the first report of an autosomal recessive anemia presenting with microcytic hypochromia, abnormal $\mathrm{Hb}$ profile, and other distinctive erythrocyte phenotypes, and provides insight into the multiple roles of KLF1 during erythropoiesis.

European Journal of Human Genetics (2015) 23, 1341-1348; doi:10.1038/ejhg.2014.291; published online 14 January 2015

\section{INTRODUCTION}

A great diversity of hereditary anemia occurs in natural populations with high genetic heterogeneity. Affected individuals present with erythrocyte defects arising from genetic variations, ${ }^{1}$ including hemoglobinpathy, ${ }^{2}$ glucose-6-phosphate dehydrogenase deficiency, ${ }^{3}$ and other types of hereditary hemolytic anemia. ${ }^{4}$ Congenital dyserythropoietic anemias (CDAs) are a heterogeneous group of hereditary disorders characterized by ineffective erythropoiesis as the predominant cause of anemia. Originally, three major types (CDA I, II, and III) were recognized based on characteristic morphological aberrations. ${ }^{5,6}$ The first of these to be described genetically was CDA I, ${ }^{7}$ for which $\sim 60 \%$ of patients have bone marrow abnormalities resulting from mutations in the codanin-1 gene $(C D A N 1){ }^{6}$ C15ORF41 was later identified as another cause of CDA I in pedigrees that were negative for CDAN1 mutations. ${ }^{8}$ Recent studies have attributed CDAII and CDAIII to mutations in SEC23B and KIF23, respectively. ${ }^{9}, 10$

A dominant mutation in the gene encoding the Krüppel-like factor 1 (KLF1) has been identified as the cause of the novel type IV CDA. Interestingly, all four documented cases were sporadic and carried the same de novo mutation c.973 A>G (p.(Glu325Lys)) in the second zinc finger (ZF) domain of KLF1. CDA IV combines features of hemoglobinopathy, red blood cell (RBC) membrane defects, and hereditary persistence of fetal hemoglobin (HPFH), which are not seen in the other types of CDA. ${ }^{11,12}$ However, various mutations in human KLF1 have been described that are associated with benign phenotypes. Compound heterozygotes for KLF1 mutations are associated with both benign and disease phenotypes. In one Sardinian family, compound heterozygosity for mutations in KLF1 was associated with mild anemia and increased fetal hemoglobin (HbF) and $\mathrm{RBC}$ protoporphyrin, ${ }^{13}$ and a recent report described eight compound heterozygotes for mutations in KLF1 among patients presenting with severe, transfusion-dependent hemolytic anemia known as chronic nonspherocytic hemolytic anemia (CNSHA). In that study, most of the cases had co-inherited $\alpha$ or $\beta$ thalassemia mutations and it was therefore not possible to clearly discern their hematological status. ${ }^{14}$ Studies suggest that the amount and type of mutant KLF1 protein produced are responsible for different RBC phenotypes. ${ }^{1-18}$ Further research is required to determine the relationship between the various KLF1 mutations and the severity of clinical phenotypes.

Here, we report a thalassemia-like syndrome in two Chinese male children diagnosed with thalassemia intermedia prior to their arrival at our clinic. A set of 64 genes involved in hemoglobin $(\mathrm{Hb})$ regulation and inherited hemolytic anemia was analyzed, and KLF1 mutations were identified in two patients. The impact of these mutations on the transcriptional activity of KLF1 was evaluated with in vitro promoterreporter assays. The results showed that compound heterozygous

${ }^{1}$ Department of Medical Genetics, School of Basic Medical Sciences, Southern Medical University, Guangzhou, China; ${ }^{2}$ Department of Hematology, 303rd Hospital of the People's Liberation Army, Nanning, China

${ }^{3}$ These authors contributed equally to this work

*Correspondence: Dr X Xu, Department of Medical Genetics, School of Basic Medical Sciences, Southern Medical University, Guangzhou 510515, Guangdong, China. E-mail: gzxuxm@pub.guangzhou.gd.cn

Received 12 March 2014; revised 14 November 2014; accepted 9 December 2014; published online 14 January 2015 
mutations that disrupt the ZF domain of KLF1 leads to an autosomal recessive syndrome of congenital microcytic hypochromic anemia. Data on the clinical characteristics and differential diagnoses of this disease are also presented.

\section{MATERIALS AND METHODS}

\section{Patients}

The two patients and their eight relatives were from two unrelated Chinese families. Both patients had nonconsanguineous parents. The two affected children, both male (Patient JK (LOVD id: \#0002183), 4-year-old and Patient GH (LOVD id: \#0002184), 12-year-old), had similar disease histories. Both had experienced hyperbilirubinemia in their early years, attributable to hemolytic anemia, and both had been diagnosed with thalassemia intermedia by the age of 6 months based on their abnormal $\mathrm{Hb}$ profiles and the hematological features of microcytic hypochromic anemia. GH was receiving regular transfusions every 3 months, usually maintaining an $\mathrm{Hb}$ concentration above $90 \mathrm{~g} / \mathrm{l}$. The patient's severe iron overload, with a ferritin level of $>2000 \mathrm{ng} / \mathrm{ml}$, was attributed to prolonged transfusion with irregular iron-chelation therapy. As a result, splenomegaly was much more severe in GH than in JK, who underwent blood transfusions only once during a relatively short illnesses, and showed a modestly elevated ferritin level of $396 \mathrm{ng} / \mathrm{ml}$. Upon examination, the two patients were pale, with no thalassemic facies. This study was approved by the local research ethics committee. Written informed consent was obtained from the participants.

\section{Hematological and biochemical tests}

Hematological parameters and globin chain levels were evaluated as previously described. ${ }^{19,20}$ The $\zeta$ globin chain level was determined using a previously described method. ${ }^{21}$ The expression of cluster of differentiation (CD) 44 in mature erythrocytes was assessed by flow cytometry. Blood groups were typed with the ID-Micro Typing System (Bio-Rad, Hercules, CA, USA) using an indirect antiglobulin gel test, with quality-controlled sera directed against Lua and Lub obtained from the National Rare Blood Bank of China.

\section{Morphological and ultrastructural analyses}

Bone marrow samples were collected by aspiration from the patients as well as from the father of $\mathrm{GH}$, who was a KLF1 mutation carrier as a control. Samples were fixed in $2.5 \%$ glutaraldehyde in $0.1 \mathrm{M}$ phosphate buffer, postfixed in $2 \%$ osmium tetroxide in phosphate-buffered saline, dehydrated in ethanol, and embedded in paraffin, then examined by transmission electron microscopy (TEM) using a JEM-1400 instrument (JEOL, Tokyo, Japan).

\section{Molecular genetic tests}

DNA sequencing was used to exclude mutations at the $H B A$ and $H B B$ loci and causative mutations in the $C D A N 1,{ }^{7}$ C15ORF $4,{ }^{8}$ SEC $23 B$ 1, ${ }^{9}$ and KIF23 genes. ${ }^{10}$ Deletional $\alpha, \beta$, and $\delta \beta$-thalassemia were excluded by multiplex ligationdependent probe amplification (MRC-Holland, Amsterdam, The Netherlands). Next-generation sequencing was used to sequence a set of 64 genes that are involved in $\mathrm{Hb}$ regulation and inherited hemolytic anemia, including the KLF1 gene (summarized in Supplementary Methods). The KLF1 mutations NM_006563.3:c.892 G >C and NM_006563.3:c.1012C > T and associated phenotypes have been submitted to the Locus-Specific Mutation Databases (dbSNP with ID ss825692192 and ss825692193) and the gene variant database (www.LOVD.nl/KLF1). Candidate variants associated with elevated $\mathrm{HbF}$ levels, including rs7482144 (Chr11.hg19:g.5254939 G > A), rs766432 (Chr2. hg19:g.60492835C > A), rs11886868 (Chr2.hg19:g.60493111C > T), rs9399137 (Chr6.hg19: g.135097880 T >C), and rs66650371 (Chr6.hg19:g.135097495_ 135097497delTAC) were confirmed by DNA sequencing.

The alignment of KLF1 missense mutations was analyzed by sorting intolerant from tolerant substitutions, and the functionality of KLF1 missense mutations was predicted with Polymorphism Phenotyping. Homology models were constructed based on the crystal structure of a KLF4-DNA complex (Protein Data Bank ID: 2WBU). The amino-acid sequence identity between KLF1 and KLF4 is $84 \%$, which have high similarity in the DNA-binding domain. Initial models of c.892 G > C (p.(Ala298Pro)) and c.1012C > T (p.(Pro338Ser)) were created by manually mutating amino-acid and DNA residues.

\section{Functional analysis}

K-562 and HEK-293 cells were cultured in RPMI 1640 medium with 10\% fetal bovine serum (Life Technologies, Carlsbad, CA, USA) and appropriate antibiotics, according to standard protocols. Full-length wild-type (WT) and mutant KLF1 open reading frames were cloned into the pEGFP-C1 vector (BD Biosciences, San Jose, CA, USA). Protein expression was determined by western blotting using a mouse anti-human KLF1 polyclonal antibody (Abcam, Cambridge, UK), with $\beta$-actin used as a loading control. Promoter-luciferase (Luc) reporters for $H B B, B C L 11 A$, and CD44 were constructed using pGL4.12 (Promega, Madison, WI, USA) (Supplementary Table S1 and Supplementary Figure S1). K-562 cells were co-transfected with a given promoter-Luc reporter (300 ng) along with pSV- $\beta$-galactosidase plasmid (200 ng) to normalize transfection efficiency and either WT or mutant KLF1 (300 ng) or both KLF1 constructs (150 ng each).

\section{RESULTS}

\section{Analysis of hemoglobinopathies}

Correlations between genotypes and phenotypes specific to hemoglobinopathies were analyzed according to the clinical presentation of the two patients with thalassemia intermedia. The patients had the hematological features of thalassemia, including microcytic hypochromic anemia, manifesting as increased $\mathrm{HbF}$, elevated reticulocyte count, and slightly increased $\mathrm{Hb}$ Bart's level (Supplementary Figure S2). Abnormalities in peripheral erythrocytes and bone marrow erythroblasts were also detected (Figures 1a-c). A marked increase in $\gamma$-globin and a mild-to-moderate reduction in $\beta$-globin chains were observed by analyzing hemolysates from patients' peripheral blood (Figure 1d). However, mutations in the $\alpha$-, $\beta$-, or $\gamma$-globin genes that could cause thalassemic phenotypes similar to those seen in the patients were excluded, prompting a screening of 64 genes involved in $\mathrm{Hb}$ regulation and inherited hemolytic anemia that could be responsible for the observed phenotypes.

\section{Identification of $K L F 1$ variants}

Next-generation sequencing of 64 candidate genes revealed compound heterozygous KLF1 mutations in the patients, in addition to the known variant rs2072597 Chr19.hg19:g.12885926 T >C of this gene. A frameshift mutation, c.525_526insCGGCGCC (p.(Gly176ArgfsTer179)), and one of two missense mutations, c.892 G >C (p.(Ala298Pro)) and c.1012C > T (p.(Pro338Ser)), were confirmed by Sanger sequencing of the patients (Supplementary Figure S3), and healthy family members from the two unrelated Chinese families. A pedigree analysis showed a recessive pattern of inheritance for the disease in both families (Figure 2a). The two missense mutations in KLF1 were absent in control chromosomes from 400 normal individuals who were included in the analysis. KLF1 has been considered as a causative gene of CDA. We followed this clue to determine the relationship between this thalassemia-like syndrome and CDA. To exclude the possibility that other known CDA genes were responsible for the thalassemia-like syndrome in the two patients, the CDAN1, SEC23B, C15ORF41, and KIF23 genes were also screened for mutations, but none were detected by direct DNA sequence analysis (data not shown). Five known variants associated with elevated HbF were genotyped (Supplementary Table S2), but the outcomes could not explain the observed increases of $26.3 \%$ (Patient $\mathrm{JK}$ ) and $33.2 \%$ (Patient GH) in HbF. Multiple alignment of KLF1 from various mammalian species showed conserved Ala and Pro at residues 298 and 338, respectively (Figure 2b). Three types of mutations were predicted to disrupt the ZF domain of KLF1: c.525_526insCGGCGCC p.(Gly176ArgfTer179) generated a truncated protein lacking the ZF domain; c.892G > C p.(Ala298Pro) in the first ZF domain; and c.1012C $>\mathrm{T}$ p.(Pro338Ser) located between the 

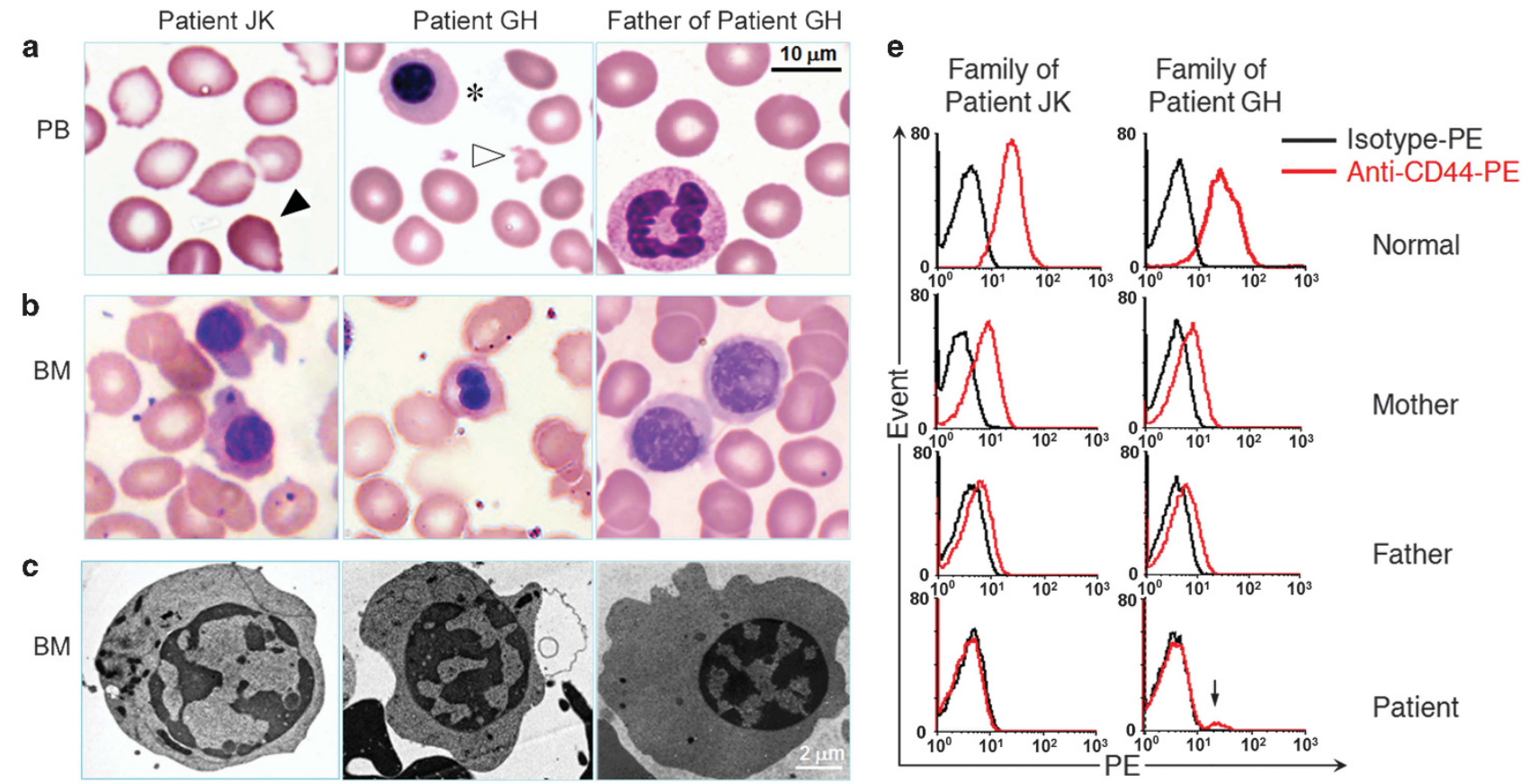

Mother

d

Patient JK

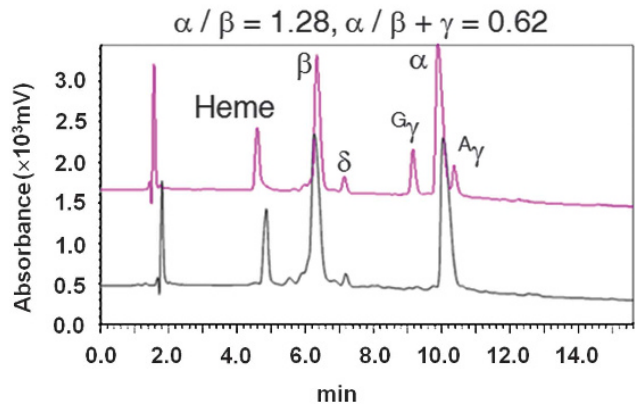

Patient GH

$\alpha / \beta=1.17, \alpha / \beta+\gamma=0.68$

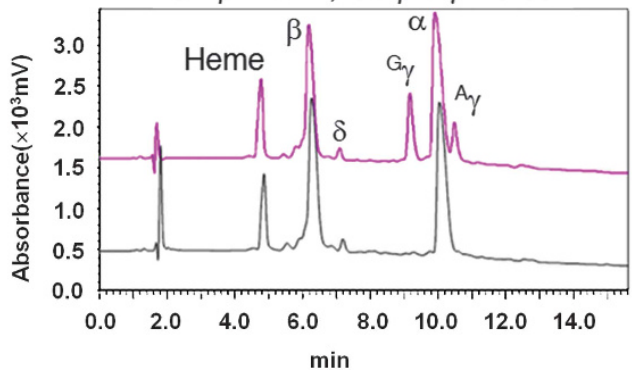

Figure 1 Phenotypic analyses of the patients in this study. (a) Peripheral blood smears from patients JK and GH show tear drop-shaped poikilocytes (black arrow), microcytosis and hypochromasia, fragmented erythrocytes (white arrow), and nucleated erythroblasts (asterisks). (b) Bone marrow smears show poikiloblast. (c) TEM analysis shows erythroblasts with heterochromatin clumps around the nuclear periphery. (d) Reversed-phase high-performance liquid chromatographic analysis of globin chains in JK, GH, and a healthy individual. Peaks and corresponding retention times for heme groups and different globin chains are indicated (red, patient; black, control). Data for $\alpha / \beta$ and $\alpha / \beta+\gamma$ ratios are shown above the chromatograms. (e) Flow cytometric analysis of CD44 expression in mature erythrocytes shows erythroid-specific CD44 deficiency in patients and reduced expression in their heterozygous parents. The mixed phenotype with low CD44 expression (arrow) likely arose from still-circulating transfused blood (50 days after the last transfusion).

second and third ZF domains (Figure 2c). Comparative structural homology modeling of the c.892G $>$ C p.(Ala298Pro) and c.1012C > T p.(Pro338Ser) mutations revealed how they affected the binding activity of the highly conserved ZF domain: an Ala-to-Pro substitution at position 298 perturbed the network of hydrogen bonds formed by S294, A298, and R301, and undermined the conformational stability of the ZF1 $\alpha$-helix by restricting the flexibility of the loop. The substitution of Pro for Ser at position 338 changed the conformation of the TGERP-like linker between ZF2 and ZF3 by relaxing the secondary structure via disruption of the predicted hydrogen bonds between R337 to A347 (Figure 2d).

\section{Unique phenotypic patient characteristics}

To further demonstrate the effects of KLF1 variants on various phenotypic changes of erythroid cells, apart from the morphological analysis of peripheral erythrocytes and bone marrow erythroblasts (Figures 1a and b). Clinical and laboratory data for affected and heterozygous members of the two Chinese families are provided in Table 1. The hematological parameters and $\mathrm{Hb}$ profiles of these patients were more complex than those of thalassemia patients. Examination of bone marrow smears revealed poikiloblast (Figure 1b) accompanied by erythroid hyperplasia (JK, 51.5\% and $\mathrm{GH}, 61 \%)$. The TEM analysis showed an abnormal heterochromatin organization with heterochromatin clumps around the nuclear periphery (Figure 1c). The patients presented with moderate anemia combined with a wide spectrum of erythroid-specific defects, such as microcytosis/hypochromia with an $\operatorname{In}[\mathrm{Lu}]$ blood type and reduced expression of CD44 protein. However, all eight adult carriers from the two Chinese families were asymptomatic, although they displayed a few specific benign phenotypes, including the $\operatorname{In}[\mathrm{Lu}]$ blood type and moderately reduced expression of CD44 protein (Table 1 and Figure 1e). In summary, three main types of phenotypic abnormality were linked to this disorder: microcytic hypochromic anemia with a high reticulocyte count, decreased $\beta / \alpha$ ratio, and mildly increased $\mathrm{Hb}$ 


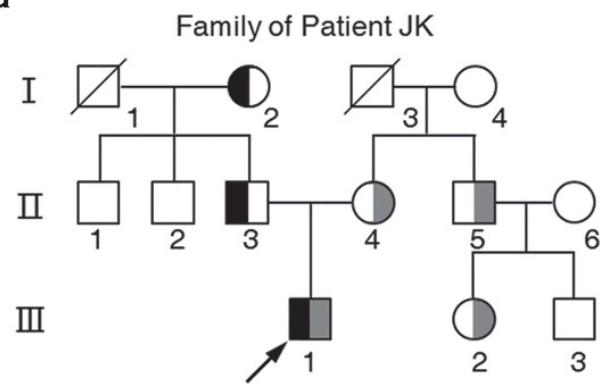

Family of Patient GH

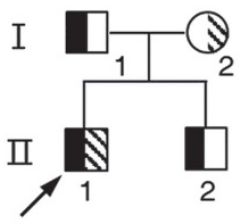

C

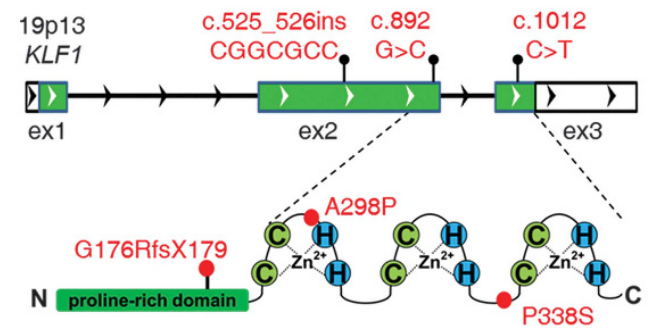

d

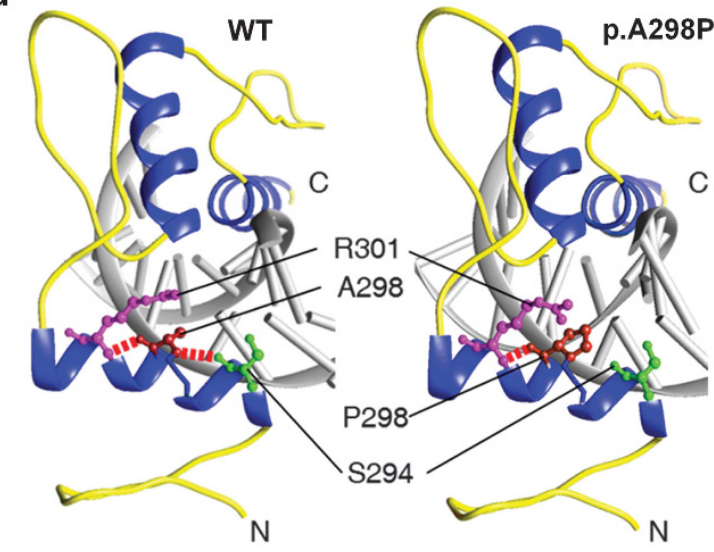

rabbit QAAHACAHPGCGKSYTKSSHLKAHLRTHTGEKP mouse QAAHTCGHEGCGKSYTKS SHLKAHLRTHTGEKP

rat QAAHTCGHEGCGKSYT KS SHLKAHLRTHTGEKP

chicken TATHNCEYPGCGK T YTKSSHLKAHMRTHTGEKP zebraf ish MATHTCTYAGCGKT Y TKSSHLKAHHRTHTGEKP

P338S H T GQRSFR C Q L C PRA F S R D H L A LHMKRHL

human H TGQRPFR C Q L C PRA F S R S D HL A LHMKRHL

monkey HTQRPFRCQLCPRAFSRSDHLALHMKRHL

cow HTQRPFRCQLCSRAFSRSDHLALHMKRHL

pig TGQRPFRCQLCPRAFSRSDHLALHMKRHL

$\operatorname{dog}$ TGQRPFRCQLCPRAFSRSDHLALHMKRHL

rabbit HTGQRPFRCQFCPRT FSRSDHLALHMKRHL

mouse H TGHRPCCGLCPRA F S RSDHLALHMKRHL

rat $\mathrm{TGHRPFCCGLCPRAFSRSDHLALHMKRHL}$

chicken HTGHPFQCHLCERAFSRSDHLALHMKRHM

zebrafish

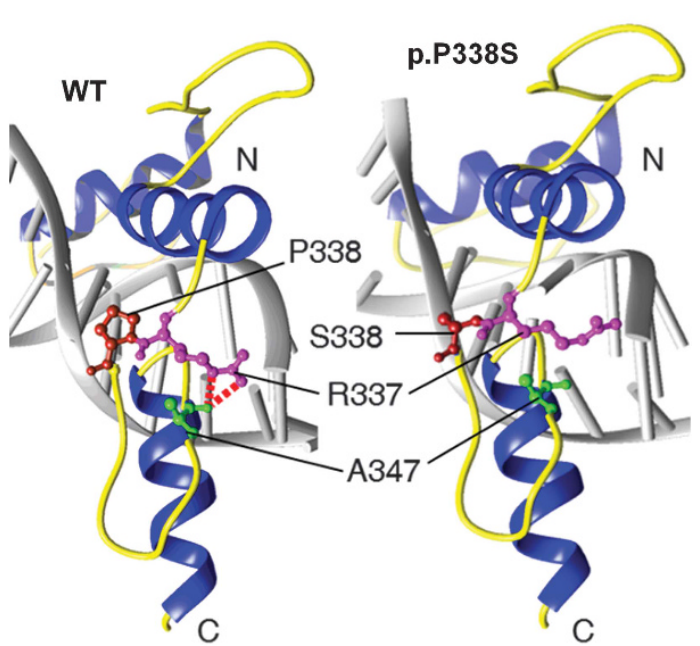

Figure 2 Identification of disease-associated KLF1 mutations. (a) Pedigrees for two unrelated Chinese families and identified KLF1 mutations. Representative chromatograms show KLF1 sequences in probands' genomic DNA. Amino acids are indicated above each chromatogram, with base/amino-acid changes highlighted in red. (b) Sequence alignment of KLF1 proteins of various mammalian species shows a conserved Ala at residue 298 (upper red box) in the ZF1 domain and Pro at residue 338 (lower red box) in the TGERP-like linker between ZF2 and ZF3. (c) The KLF1 gene (NG_013087.1) and its products, consisting of three exons (green boxes, coding regions; white boxes, untranslated regions). The gene product consists of an N-terminal Pro-rich transactivation domain (green box) and three C-terminal $\mathrm{C} 2 \mathrm{H} 2 \mathrm{ZF}$ domains. Base alterations and amino-acid substitutions corresponding to mutations are shown in red. (d) Upper panel: steady-state network of hydrogen bonds (broken red lines) formed by A298 (red), S294 (green), and R301 (purple) in WT KLF1 (left) and the unstable $\alpha$-helix in the mutant A298P (right). Lower panel: predicted hydrogen bonding (broken red lines) of R337 (purple) to A347 (green) in WT KLF1 (left) and the loss of hydrogen bonds in the mutant P338S (right). DNA is shown in gray.

Bart's level; unusual benign changes in erythrocytes, including the InLu phenotype, abolition of the RBC membrane adhesion molecule CD44, moderately increased RBC protoporphyrin and high levels of $\zeta$-globin chains; and morphological abnormalities, including poikiloblast and abnormal heterochromatin organization.

\section{Altered localization of mutant KLF1}

To study protein localization, we examined the expression subcellular localization of WT KLF1 and three KLF1 mutants using a GFP-tagged subcellular localization assay. The nuclear localization of the three mutants is not affected as seen in Figure 3a. Furthermore, it is not recognized by the protein quality control of the cell and degraded as seen in the western blot (Figure 3b). Interestingly, the c.525_526insCGGCGCC (p.(Gly176ArgfsTer179)) mutation, which was similar in size to the WT KLF1 (only nine amino acids shorter), showed an additional feature, namely this mutant seems to produce aggregates in the nucleus, which may contribute to the phenotype by inducing stress in cells due to the accumulation of misfolded proteins. 


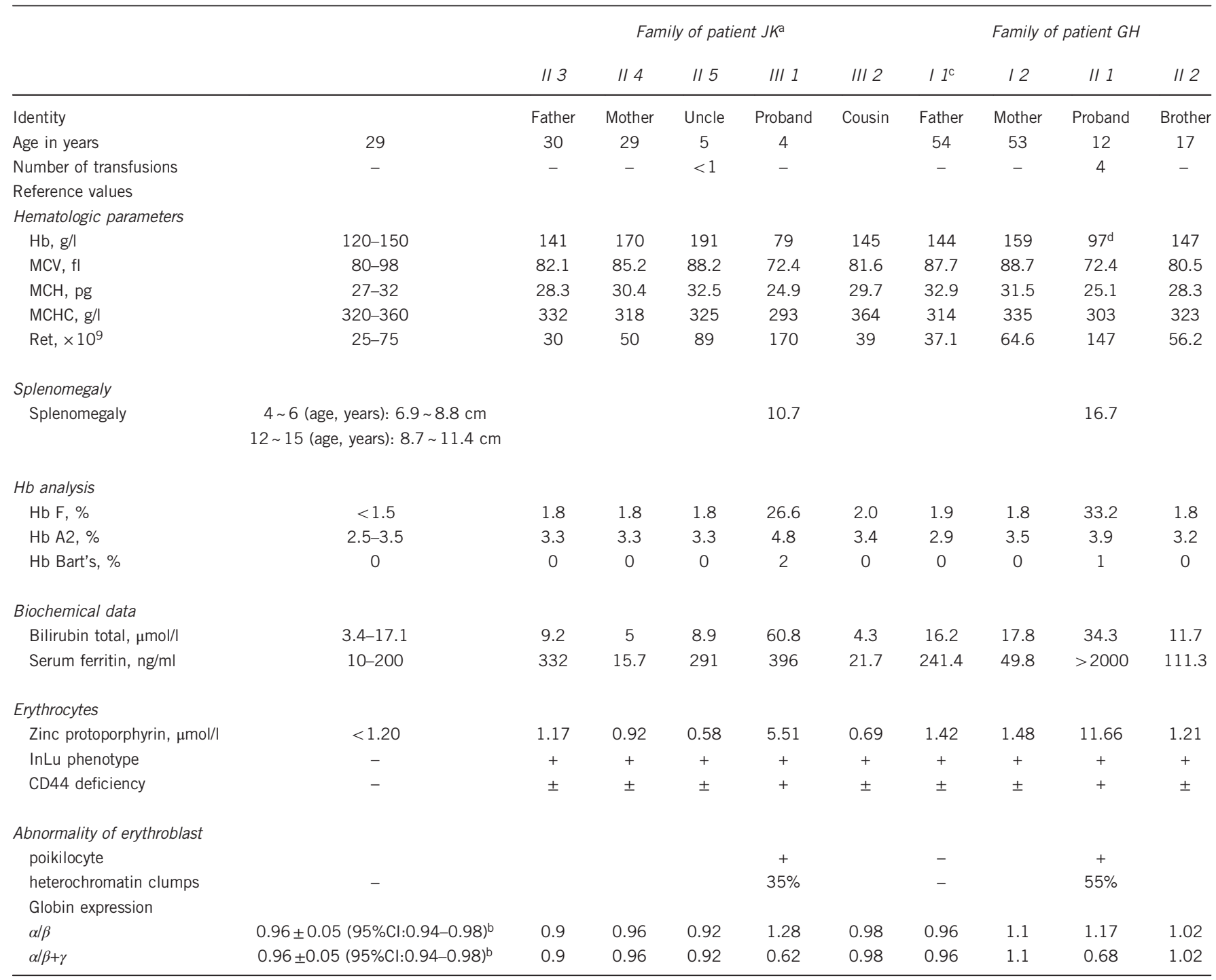

+ and - indicate positive and negative phenotypes described in rows, \pm indicates the expression is lower than normal individuals.

aFour family members in family A without KLF1 mutation had normal hematologic parameters with $\mathrm{Hb} F$ between $1.3 \%$ and $1.7 \%$.

aFour family members in family A without KLFI mutation had normal
bThirty healthy individuals were analyzed and used as normal control.

cUltrastructural analysis shows normal erythropoiesis and served as a control.

dIn order to maintain $\mathrm{Hb}$ level of $90 \mathrm{~g} / \mathrm{l}$ to sustain the growth and development, regular transfusion was given after diagnosis at the age of 6 months.

Hence, this protein aggregation, which probably escapes nonsensemediated mRNA decay, may indeed contribute to the disease mechanism of this mutant.

\section{Effect of KLF1 mutations on HBB, CD44, and BCL11A transcription}

It was reasoned that abnormal erythroblast phenotypes resulted from the loss of KLF1 function. Thus, the transcriptional activity of KLF1 was investigated in $H B B, C D 44$, and BCL11A, three KLF1 target genes associated with disease phenotypes.

In the KLF1-tagged promoter-reporter assay, KLF1 mutants reduce the transcription of $H B B, C D 44$, and BCL11A in a simulated compound heterozygote for two of the mutations or a homozygote for each of the three mutations. The mutations did not interfere with the transcriptional activity of WT KLF1, and compound mutants had the same degree of reduction in reporter activity (26-61\%) as single mutants. As expected, the c.525_526insCGGCGCC (p.(Gly176ArgfsTer179)) mutant had the most prominent loss of transcriptional activity compared with the WT. However, when WT KLF1 was coexpressed with each of the three variants, the simulated heterozygotes were still able to activate the target gene promoters to varying degrees (Figures 3c-e): the transactivation decreased to only $85-94 \%$ for $H B B$ and $B C L 11 A$, and up to $42-50 \%$ for $C D 44$, respectively. This suggests that two mutant KLF1 alleles can differentially affect target genes to produce distinct phenotypes; for instance, the downregulation of $H B B$, $B C L 11 A$, and CD44 expression leads to an $\alpha /$ non- $\alpha$ chain imbalance, $\mathrm{HPFH}$, and $\mathrm{In}[\mathrm{Lu}]$ blood type, respectively.

\section{DISCUSSION}

This study elucidated a new cause of congenital microcytic hypochromic anemia in pediatric patients displaying a range of RBC phenotypes. The patients' autosomal recessive blood disorder was associated 


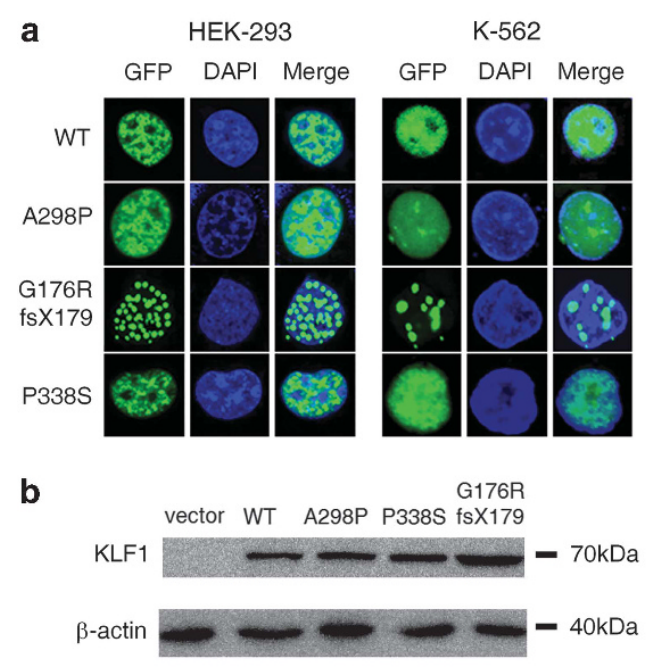

d
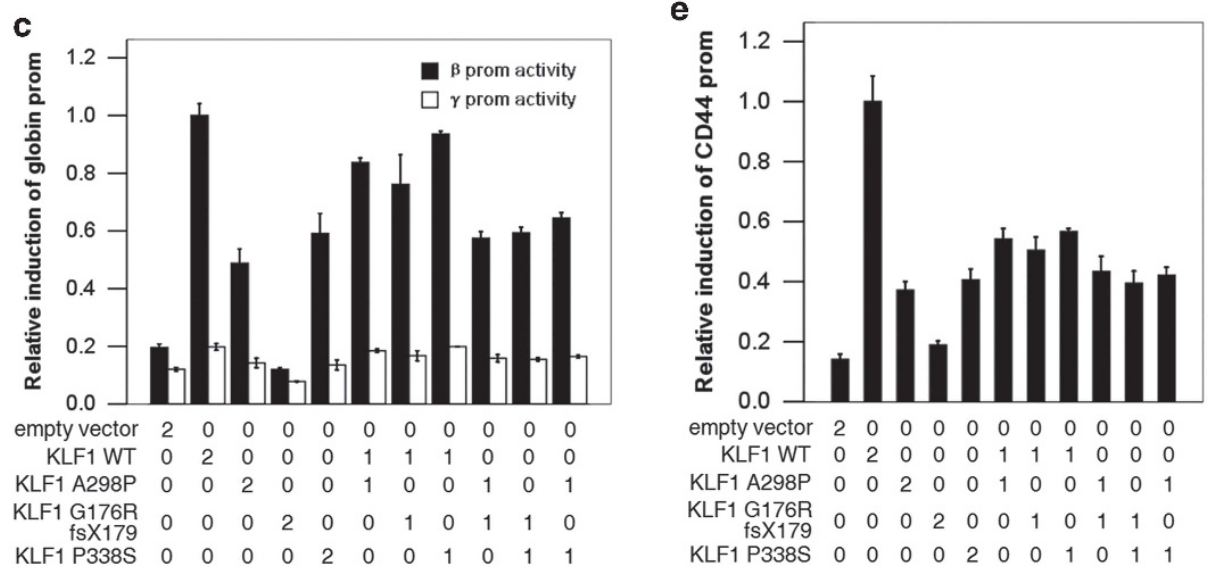

Figure 3 Effect of mutations on KLF1 function. (a) Subcellular localization of constructs encoding GFP-tagged wild-type KLF1 (WT) and the three mutants in HEK-293 and K-562 cells. Confocal images of GFP (green), DAPI nuclear staining (blue), and the merged signals are shown. (b) Expression of WT KLF1 and three mutants in K-562 cells, as determined by western blotting. (c-e) Effect of KLF1 mutations on the regulation of KLF1 target genes HBB, BCL11A, and CD44 in K-562 cells co-transfected with a HBB, BCL11A, or CD44 promoter-Luc construct, along with KLF1 constructs or an empty vector at concentrations of 0,150 , or $300 \mathrm{ng}(0,1$, and 2, respectively). Data in (c-e) represent mean \pm s.d. of three independent experiments.

with non-transfusion-dependent anemia, and showed the slight morphological abnormalities in erythroblasts, abnormal globin synthesis, RBC benign changes (including the benign $\operatorname{In}[\mathrm{Lu}]$ blood type and CD44 deficiency and increased RBC protoporphyrin), red cell microcytosis, and hypochromia with elevated $\mathrm{HbF}$ and $\zeta$ globin chains. Both patients were compound heterozygotes for frameshift mutation c.525_526insCGGCGCC (p.(Gly176ArgfsTer179)) and one of two missense mutations c.892G $>\mathrm{C}$ (p.(Ala298Pro)) and c.1012C $>$ T (p.(Pro338Ser)) in KLF1, providing an explanation for the phenotypic similarities of the two patients. KLF1 mutations can lead to loss or gain-of-function, giving rise to distinct phenotypic states, including CDA IV, CNSHA, and mild anemia, and induce increases in $\mathrm{HbF}$ level. ${ }^{12-14}$ In contrast, the patients in this study exhibited the thalassemia phenotypes and unusual changes associated with KLF1 loss-of-function mutations. For instance, the microcytic hypochromic anemia with poikiloblast and abnormal heterochromatin organization in erythroblasts are not observed in CDA IV. In addition, unlike CNSHA, microcytic hypochromic anemia presents thalassemia phenotypes that are not linked to globin genes.

Notable features of this disease were RBC hypochromia and microcytosis, which could largely be explained by reduced synthesis of $\mathrm{HbA}$, resulting in smaller ethythrocytes containing lower amounts of $\mathrm{Hb}^{22}$ This was evidenced by the reduction in total $\beta$-chain synthesis in the KLF1 promoter-reporter assay. The $\beta$-globin promoter is transactivated by KLF1; the patients in this study had moderate levels of $\beta$-chain synthesis. However, KLF1 inhibits $\gamma$-globin expression by transactivating $B C L 11 A .^{23}$ In the present reporter assays in K-562 cells, the transcriptional activities of the $\gamma$-globin promoter were unaffected, because these cells do not express BCL11A. High levels of zinc protoporphyrin were present only in compound heterozygotes with two KLF1 mutations in trans, as previously described in a Sardinian HPFH family, ${ }^{13}$ although the combination of mutations in this study differed from the Sardinian variants c.809C $>$ A (p.(Ser270Ter)) and c.994A > C (p.(Lys332Gln)). Surprisingly, compound heterozygotes of the KLF1 mutations c.809C>A (p.(Ser270Ter)) and c.994A >C (p.(Lys332Gln)), both of which affect the ZF domain, did not produce severe anemia or a $\beta$-thalassemia phenotype, suggesting that the c.994A > C (p.(Lys332Gln)) mutation has little impact on the transactivation capacity of KLF1. ${ }^{13,17}$ Furthermore, the HS2 $-\gamma$ prom/ Renilla $-\beta$ prom/Firefly reporter assay demonstrated that the compound c.994A > C (p.(Lys332Gln)) mutations in the Sardinian family had less effect than the mutations described in the present study. (Supplementary Figure S4). Only moderately elevated $\mathrm{HbF}$ (9.5\%) was observed in an adult Vietnamese patient who was a compound 
Table 2 Characteristic features of the reported anemia, HPFH, CDA IV, and thalassemia intermediate

\begin{tabular}{|c|c|c|c|c|}
\hline Identity & HPFH & $\begin{array}{l}\text { Thalassemia } \\
\text { intermediate }\end{array}$ & CDA IV & $\begin{array}{c}\text { Reported } \\
\text { anemia }\end{array}$ \\
\hline Anemia & $\mathrm{n}$ & +++ & +++ & +++ \\
\hline Microcystis/hypochromia & + & +++ & $\mathrm{n}$ & ++ \\
\hline Reticulocytes & $n$ & +++ & + & ++ \\
\hline \multicolumn{5}{|l|}{ Erythrocytes } \\
\hline Zinc protoporphyrin & $\mathrm{n}$ & $n$ & NA & $\uparrow \uparrow$ \\
\hline In(Lu)phenotype & - & - & $\mathrm{n}$ & + \\
\hline CD44 deficiency & - & - & + & + \\
\hline$\zeta$-globn & - & - & + & + \\
\hline \multicolumn{5}{|l|}{ Morphology } \\
\hline poikilocyte & - & + & NA & + \\
\hline heterochromatin clumps & - & - & - & +++ \\
\hline \multicolumn{5}{|l|}{ Hb expression } \\
\hline $\mathrm{HbF}$ & $\uparrow \uparrow \uparrow$ & $\uparrow \uparrow$ & $\uparrow \uparrow$ & $\uparrow \uparrow$ \\
\hline$\alpha / \beta$ & $\uparrow \uparrow \uparrow \uparrow$ & $\uparrow \uparrow \uparrow \uparrow$ & $\uparrow \uparrow \uparrow$ & $\uparrow \uparrow$ \\
\hline$\alpha / \beta+\gamma$ & $\uparrow$ & $\uparrow \uparrow \uparrow$ & $\uparrow \uparrow$ & $\downarrow$ \\
\hline Gene & $H B B$ & $H B B$ & $K L F 1$ & $K L F 1$ \\
\hline Inheritance & $A R$ & AR & De novo & AR \\
\hline
\end{tabular}

Abbreviation: NA: not available. + and - indicate positive and negative phenotypes described in the columns. $\mathrm{n}$ indicates normal, $\uparrow$ and $\downarrow$ indicate increase and decrease expression.

heterozygote for the c.525_526insCGGCGCC (p.(Gly176ArgfsTer179)) frameshift and c.152T $>$ G (p.(Leu51Arg)) missense mutations in the KLF1 gene. ${ }^{16}$ This indicates that an asymptomatic phenotype likely results from a single c.525_526insCGGCGCC (p.(Gly176ArgfsTer179)) mutation rather than a combination of two KLF1 mutations, as the c.152T $>\mathrm{G}$ (p.(Leu51Arg)) mutation in exon 1 of KLF1 did not alter the activity of the ZF domain. Therefore, KLF1 mutations in the ZF likely give rise to this disease.

In this study, c.525_526insCGGCGCC p.(Gly176ArgfsTer179) heterozygous carriers lacking pathological symptoms exhibited the $\mathrm{In}[\mathrm{Lu}]$ blood type and showed decreased expression of CD44 (Supplementary Table S3). Several similar cases have previously been associated with the KLF1 frameshift mutations c.569delC (p.(Pro190LeufsTer47)) $)^{24}$ and c.954dupG (p.(Arg319GlufsTer34) ${ }^{18}$. It is likely that different target genes respond to different thresholds of KLF1 activity. Although the trans activity was almost completely abolished in the c.525_526insCGGCGCC (p.(Gly176ArgfsTer179)) variant, heterozygous carriers retained one copy of the WT KLF1 gene that would express fully functional protein, albeit at a reduced dose.

Unlike for CDA IV, which is caused by a dominant KLF1 mutation or intermediate thalassemia (Table 2), the two patients had clinical and hematological features consistent with microcytic hypochromic anemia, with relatively minor morphological abnormalities, elevated reticulocyte count, $\mathrm{HPFH}$, and slightly increased $\mathrm{Hb}$ Bart's level, along with other benign phenotypes.

More than 30 different human KLF1 variants have been identified and found to be associated with altered RBC phenotypes and diseases. Interestingly, all the KLF1 mutations examined here altered the $\mathrm{ZF}$ domain and compromised the transactivation capacity of the protein. This suggests the importance of the ZF domain in regulating KLF1 target genes. We have identified here for the first time compound heterozygous KLF1 mutations responsible for microcytic hypochromic anemia. In vitro functional assays showed the additive loss-of-function effects of these mutations, which caused a reduction in the expression of the $H B B, B C L 11 A$, and $C D 44$ genes involved in erythropoiesis. This resulted in a phenotype that included minor morphological abnormalities in erythroblasts, $\beta$-thalassemia, RBC membrane defects, In[Lu] blood type, and increases in $\mathrm{HbF}$ and $\mathrm{RBC}$ protoporphyrin. All eight members of the two Chinese families who were mutation carriers were basically normal, although a few had benign phenotypes such as the In [Lu] blood type (Supplementary Table S3). Based on the observation that double mutants of c.525_526insCGGCGCC (p.(Gly176ArgfsTer179)) and c.892G > C (p.(Ala298Pro)) or c.1012C > T (p.(Pro338Ser)) affected the ZF domain in trans, it was concluded that the composite phenotypes observed in these patients could be produced by interplay between the KLF1 rest function, transcriptional activity of mutants, and the sensitivity of target promoter to KLF1 transcriptional activity. Thus, KLF1 is the first gene to be implicated in this disorder.

This study identified three recessive mutations in the KLF1 gene, all of which altered the ZF domain of KLF1; the effect of genetic heterogeneity on phenotypic outcome provides new insight into the multiple roles of KLF1 during erythropoiesis. The rate of these mutations in the Chinese population was found to be $\sim 15$ per 1000 individuals, with the c.525_526insCGGCGCC (p.(Gly176ArgfsTer179)) frameshift being the most common. ${ }^{25}$ This suggests that autosomal recessive anemia is more widespread than is currently reported based on the frequency of KLF1 mutations.

\section{CONFLICT OF INTEREST}

The authors declare no conflict of interest.

\section{ACKNOWLEDGEMENTS}

We thank all members of the two families and acknowledge collaboration with Dongguan Maternity and Child Care Hospital, Guangdong, China. We are especially grateful to Dr Tim M Townes for providing plasmids. This work was supported in part by the National Natural Science Foundation of ChinaGuangdong Joint Fund (NO. U1201222); the National Key Technology R\&D Program (No.2012BAI09B01) and the National Natural Science Foundation (No.81300378).

\section{AUTHOR CONTRIBUTIONS}

$\mathrm{JH}$ and XX designed the study; XZ, XW, and FX collected the clinical data; $\mathrm{JH}, \mathrm{DL}$, and LY performed variant analyses and expression studies; DL, JH, and XS did functional assays. XX, XZ, JH, and XS analyzed and interpreted the data; $\mathrm{JH}$ wrote paper and $\mathrm{XX}$ revised the paper.

1 Alaarg A, Schiffelers RM, van Solinge WW, van Wijk R: Red blood cell vesiculation in hereditary hemolytic anemia. Front Physiol 2013; 4: 365

2 Higgs DR, Engel JD, Stamatoyannopoulos G: Thalassaemia. Lancet 2012; 379. 373-383

3 Cappellini MD, Fiorelli G: Glucose-6-phosphate dehydrogenase deficiency. Lancet 2008; 371: 64-74.

4 Jung HL: A new paradigm in the diagnosis of hereditary hemolytic anemia. Blood Res 2013; 48: 237-239.

5 Iolascon A, Russo R, Delaunay J: Congenital dyserythropoietic anemias. Curr Opin Hematol 2011; 18: 146-151.

6 Iolascon A, Esposito MR, Russo R: Clinical aspects and pathogenesis of congenital dyserythropoietic anemias: from morphology to molecular approach. Haematologica 2012; 97: 1786-1794.

7 Renella R, Roberts NA, Brown JM et al: Codanin-1 mutations in congenital dyserythropoietic anemia type 1 affect HP1\{alpha\} localization in erythroblasts. Blood 2011; 117: 6928-6938.

8 Babbs C, Roberts NA, Sanchez-Pulido L et al: Homozygous mutations in a predicted endonuclease are a novel cause of congenital dyserythropoietic anemia type I. Haematologica 2013; 98: 1383-1387.

9 Schwarz K, Iolascon A, Verissimo F et al: Mutations affecting the secretory COPII coat component SEC23B cause congenital dyserythropoietic anemia type II. Nat Genet 2009; 41: 936-940. 
10 Liljeholm M, Irvine AF, Vikberg AL et al: Congenital dyserythropoietic anemia type III (CDA III) is caused by a mutation in kinesin family member, KIF23. Blood 2013; 121 4791-4799.

11 Jaffray JA, Mitchell WB, Gnanapragasam MN et al: Erythroid transcription factor EKLF/ KLF1 mutation causing congenital dyserythropoietic anemia type IV in a patient of Taiwanese origin: review of all reported cases and development of a clinical diagnostic paradigm. Blood Cells Mol Dis 2013; 51: 71-75.

12 Arnaud L, Saison C, Helias V et al: A dominant mutation in the gene encoding the erythroid transcription factor KLF1 causes a congenital dyserythropoietic anemia. Am J Hum Genet 2010; 87: 721-727.

13 Satta S, Perseu L, Moi P et al: Compound heterozygosity for KLF1 mutations associated with remarkable increase of fetal hemoglobin and red cell protoporphyrin. Haematologica 2011; 96: 767-770.

14 Viprakasit V, Ekwattanakit S, Riolueang $\mathrm{S}$ et al: Mutations in Kruppel-like factor 1 cause transfusion-dependent hemolytic anemia and persistence of embryonic globin gene expression. Blood 2014; 123: 1586-1595.

15 Siatecka M, Bieker JJ: The multifunctional role of EKLF/KLF1 during erythropoiesis. Blood 2011; 118: 2044-2054.

16 Gallienne AE, Dreau HM, Schuh A, Old JM, Henderson S: Ten novel mutations in the erythroid transcription factor KLF1 gene associated with increased fetal hemoglobin levels in adults. Haematologica 2012; 97: 340-343.

17 Singleton BK, Lau W, Fairweather VS et al: Mutations in the second zinc finger of human EKLF reduce promoter affinity but give rise to benign and disease phenotypes. Blood 2011; 118: 3137-3145.
18 Helias V, Saison C, Peyrard T et al: Molecular analysis of the rare in(Lu) blood type: toward decoding the phenotypic outcome of haploinsufficiency for the transcription factor KLF1. Hum Mutat 2013; 34: 221-228.

19 Xiong $F$, Sun M, Zhang $X$ et al: Molecular epidemiological survey of haemoglobinopathies in the Guangxi Zhuang Autonomous Region of southern China. Clin Genet 2010; 78: 139-148.

20 Wan JH, Tian PL, Luo WH et al: Rapid determination of human globin chains using reversed-phase high-performance liquid chromatography. J Chromatogr B Analyt Technol Biomed Life Sci 2012; 901: 5358.

21 Tang L, Zhu P, Zhou WJ et al: Development and validation of a zeta-globin-specific ELISA for carrier screening of the (-SEA) alpha thalassaemia deletion. J Clin Pathol 2009; 62: 147-151.

22 Auer J: The formation of macrocytes and microcytes from red corpuscles in hanging drop preparation. J Exp Med 1932; 56: 551-573.

23 Sankaran VG, Menne TF, Xu J et al: Human fetal hemoglobin expression is regulated by the developmental stage-specific repressor BCL11A. Science 2008; 322 : 1839-1842.

24 Singleton BK, Frayne J, Anstee DJ: Blood group phenotypes resulting from mutations in erythroid transcription factors. Curr Opin Hematol 2012; 19: 486-493.

25 Liu D, Zhang X, Yu L et al: Erythroid Krüppel-like factor mutations are relatively more common in a thalassemia endemic region and ameliorate the clinical and hematological severity of $\beta$-thalassemia. Blood 2014; 124 : 803-811.

Supplementary Information accompanies this paper on European Journal of Human Genetics website (http://www.nature.com/ejhg) 